CARDIOVASCULAR MEDICINE

\title{
Pulmonary vein ablation for idiopathic atrial fibrillation: six month outcome of first procedure in 100 consecutive patients
}

\author{
J P Bourke, A Dunuwille, D O'Donnell, S Jamieson, S S Furniss
}

Heart 2005;91:51-57. doi: 10.1136/hrt.2003.023093

See end of article for authors' affiliations

Correspondence to: Dr John P Bourke, Department of Cardiology, Freeman Hospital,

Freeman Road, Newcastle upon Tyne NE7 7DN, UK; i.p.bourke@ncl.ac.uk

Accepted 15 July 2004
Objectives: To report six month outcome in patients undergoing their first pulmonary vein ablation procedure for idiopathic atrial fibrillation (AF) at a "non-pioneering" hospital.

Design: Prospective observational study.

Setting: Specialist electrophysiology unit at a university hospital.

Patients: The first 100 consecutive patients undergoing their first pulmonary vein catheter ablation procedure for highly symptomatic, drug resistant AF in the period 1999-2002.

Main outcome measures: Incidence of symptomatic or asymptomatic, Holter documented AF six months after ablation.

Results: Mean patient age was 52 years (range 23-73 years), mean length of AF history 53 months (range 6-180 months), mean number of antiarrhythmic drug failures was 3 (range 1-5), and 81 were men. At the time of the ablation procedure, 64 had progressed to persistent AF and 23 had increased transverse left atrial diameter. The number of pulmonary veins ablated in each patient was one in 11, two in 45 , three in 36 , and four in 8 . Six months after ablation, 55 patients were consistently in sinus rhythm, asymptomatic, and without any Holter evidence of AF. The chance of being in sinus rhythm was $73 \%$ ( 29 of 64 ) in those with paroxysmal as compared with only $45 \%$ (26 of 36 ) in those with persistent AF at the time of ablation $(p=0.01$ ). Outcome was not influenced by patient age, length of AF history, or duration of persistent AF before ablation or to left atrial dimension. Follow up was complete and no patient has died or experienced a stroke during or after ablation; nor have any developed symptoms of late pulmonary vein stenosis. However, other complications occurred during or shortly after the procedure in 12 patients, including cardiac tamponade in six.

Conclusions: In selected patients with drug resistant $A F$, focal pulmonary vein catheter ablation offers a realistic prospect of achieving stable sinus rhythm compared with alternatives. However, it is a complex form of ablation with a significant risk of serious complications. Although a new milestone in arrhythmia management, the optimum ablation technique is still evolving and any impact on the natural history of AF remains to be determined.
A trial fibrillation (AF) is the most common sustained cardiac arrhythmia. ${ }^{1}$ Despite this, and perhaps because it had long been assumed that AF would not be amenable to curative ablation, AF has been relatively neglected by cardiac electrophysiology. However, recently pioneering groups have begun to unravel the complex interaction between the extrasystoles arising predominantly from pulmonary veins, which trigger $\mathrm{AF}$, and the changes that take place as a consequence of the arrhythmia, which ultimately make it permanent..$^{2-4}$ As a result, various ablation strategies have now emerged and their feasibility and success have been confirmed by the original proponents. ${ }^{256}$ The main goal of ablation is to prevent repetitive extrasystoles, which arise from the epicardial muscle sleeves of pulmonary veins, from conducting to the body of the left atrium by delivering focal lesions near the vein ostia. ${ }^{58}$ When sustained AF has led to advanced atrial remodelling, pulmonary vein ablation may need to be supplemented by ablation lines to create conduction block at key zones to stabilise atrial electrophysiology and help maintain sinus rhythm. ${ }^{910}$ Evidence for the benefits of ablation lines in restoring sinus rhythm comes from the surgical literature, with reports that AF can be abolished in 70-99\% of cases by creating lines of conduction block in one or both atria. ${ }^{1-13}$

Despite the lack of any long term efficacy data and continuing controversy about risk-benefit considerations, catheter ablation of AF is now beginning to be performed in many hospitals worldwide. ${ }^{14-18} \mathrm{~A}$ crucial question is whether the encouraging success rates reported by pioneering groups can be achieved by second wave hospitals. The goals of this research, therefore, were, firstly, to report six month outcome from the first 100 consecutive patients who underwent their first left atrial catheter ablation for AF in the absence of identifiable heart disease at our hospital; and, secondly, to determine the impact of five variables on outcome-age, length of any AF history, whether AF was paroxysmal or persistent at time of ablation, duration of persistent AF, and left atrial size-and their implications for patient and procedure selection.

\section{METHODS}

\section{Patient selection}

Patients referred to our hospital with a history of highly symptomatic paroxysmal $\mathrm{AF}$, in the absence of identifiable heart disease, were considered for catheter ablation. They were not rejected for ablation even if they had progressed to a persistent pattern of $\mathrm{AF}$ by the time of assessment or ablation. Typically, AF had not been controlled symptomatically by trials of two antiarrhythmic drugs. Structural heart disease was excluded on the basis of history and non-invasive testing. All patients had an echocardiogram to exclude ventricular systolic dysfunction or dilatation, hypertrophy, 
and valvar disease. All had normal P waves on 12 lead ECG in sinus rhythm. Transient or systemic causes for AF were excluded by blood tests for thyroid dysfunction, systemic inflammation, or biochemical derangement. Systemic hypertension was not a contraindication to ablation once blood pressure had been controlled, but patients whose arrhythmia was considered to be caused by alcohol excess were deemed unsuitable for this procedure.

\section{Consent for procedure}

Before ablation a senior member of the team explained the procedure in detail to all patients in the outpatient context. The novelty of the technique and the fact that the procedure itself is long and arduous, has uncertain medium term results, and lacks any long term outcome data, were all emphasised. Patients were quoted a small risk of serious complications, specified as embolic stroke, cardiac tamponade, and pulmonary vein stenosis. Before agreeing to undergo the procedure, patients in persistent AF were also made aware that treatment with potent antiarrhythmic drugs and full anticoagulation would need to continue for up to three months after ablation. Consent was obtained in writing usually at a preadmission clinic in the weeks before admission. The patients included in this series, therefore, were highly motivated either by intolerable symptoms or lack of acceptance of AF and the need for chronic treatment as an alternative. Patients with frequently recurring or long lasting paroxysms and all those in persistent AF were fully anticoagulated with warfarin for a minimum of one month before ablation. Anticoagulation was discontinued five days before the procedure itself.

\section{Ablation procedure}

Procedures were performed under midazolam and fentanyl sedation and supplemental oxygen. A nurse monitored and recorded the level of consciousness, blood pressure, and oxygen saturation throughout the procedure. Under local anaesthesia, catheters were sited through the right femoral vein to the right atrium and transseptally to the left atrium. A coronary sinus catheter was sited through the right internal jugular vein to the coronary sinus for internal cardioversion and left atrial pacing. In most cases two steerable $4 \mathrm{~mm}$ tip ablation catheters was passed transseptally, one through a sheath and the other through the same septal puncture without a sheath. The right atrial catheter acted as a reference and could also be used for pacing. Navigation and ablation were guided by pulmonary vein-left atrial angiograms alone in the first half of the series and subsequently by angiography and the LocaLisa intracardiac navigation system. ${ }^{10}$ After the left atrium was accessed, patients were anticoagulated with an intravenous bolus of heparin. This was supplemented later as required to maintain an activated clotting time of greater than $200 \mathrm{~s}$.

Ablation lesions were sited inside the pulmonary veins as near the ostium as possible while retaining catheter stability, with the aim of disrupting all electrical connections between the veins and the body of the left atrium. Initially only veins exhibiting arrhythmogenic behaviour were targeted for ablation; however, in the latter part of this series, attempts were made to isolate all four veins. To facilitate this, a stored reference image of the vein being ablated at any particular time was displayed and, in the second half of the series, the combination of a reference angiogram image and the LocaLisa system allowed the ostia to be identified and navigated around more accurately. Each energy delivery was maintained for $60 \mathrm{~s}$ typically and, to reduce the risk of pulmonary vein stenosis, strictly limited to $30 \mathrm{~W}$ for left upper and 20-25 W for left lower and right pulmonary veins, and with a maximum temperature cut off setting of $50^{\circ} \mathrm{C} .{ }^{19}$
For patients in sinus rhythm at the start of the procedure, catheters were positioned initially in the left and right upper pulmonary veins and moved subsequently to other veins. If spontaneous extrasystoles occurred the veins responsible were identified and targeted for ablation first. If no extrasystoles occurred during a short period of observation, coronary sinus pacing was instituted and the left upper pulmonary vein was ablated, targeting ostial sites with pulmonary potentials closely coupled to left atrial electrograms. ${ }^{20}$ The right upper pulmonary vein was addressed in the same way during sinus rhythm. For patients in persistent $\mathrm{AF}$ at the start of the procedure, attempts were made to restore sinus rhythm with internal direct current cardioversion. If this was achieved, ablation progressed as for patients in sinus rhythm thereafter. If sinus rhythm could not be maintained, the left and right upper veins were ablated empirically, before cardioversion was repeated. After ablations in the left and right upper veins had been completed, catheters were moved to the lower veins and evidence of spontaneous or pulmonary potentials was sought. For patients continuing in $\mathrm{AF}$ an attempt was made to address all four veins comprehensively. In addition, in the later part of the series linear ablations were performed with LocaLisa guidance in patients resistant to cardioversion despite pulmonary vein ablations. Lines were sited to block conduction between the mitral annulus and left lower pulmonary vein ostium (mitral isthmus line) and between the ostia of the right and left upper veins (roof line). If, at the end of the procedure, patients continually reverted to AF after cardioversion, flecainide $(1-1.5 \mathrm{mg} / \mathrm{kg}$ ) was administered to help stabilise the atria before a final cardioversion. Although the intention was to isolate the veins targeted for ablation electrically, this was not confirmed systematically in all cases, mainly because of the proportion of patients in persistent AF for most of the ablation procedure. The end point of the procedure was dictated by a composite of considerations: successful isolation of the culprit veins or extent of ablations already sited to achieve this; patient discomfort; occurrence of or concern about possibility of complications; and total duration of the procedure. Anticoagulation was reversed with intravenous protamine at the end of the procedure in most patients.

\section{Before discharge}

On the day after the procedure, all patients had an echocardiogram recorded as baseline for further follow up and to exclude pericardial effusion, and an ECG to document atrial rhythm and, if in sinus rhythm, $\mathrm{P}$ wave morphology. All patients in persistent AF before ablation were prescribed potent antiarrhythmics on discharge-typically flecainide and a $\beta$ blocker-for at least three months and anticoagulation was recommenced. However, patients taking amiodarone were continued on this and sotalol was prescribed to those with a history of previous flecainide intolerance. Treatment was individualised for patients with a history of paroxysmal AF only, and some patients chose to have their antiarrhythmic treatment discontinued altogether after the procedure. Oral anticoagulation was restarted in all patients with persistent AF before ablation, any patient with a previous embolic event, and patients with paroxysmal AF who had been taking it before the procedure. Thus, all patients were taking either warfarin or aspirin after their procedure.

\section{Follow up}

Patients were reviewed regularly, typically at six weeks and at three, six, and 12 months after their ablation procedure. This report presents outcome data for all patients at six months after their first anti-AF ablation procedure. Outcome at six 


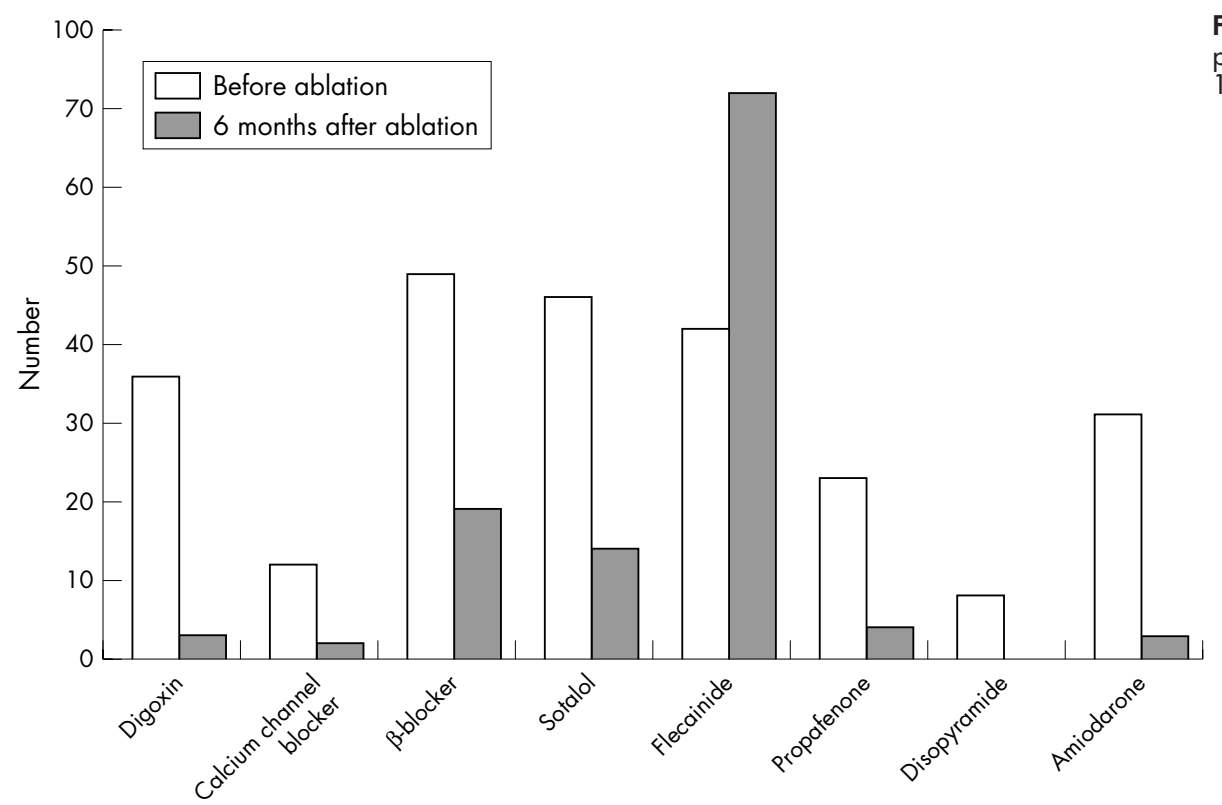

Figure 1 Antiarrhythmic drug prescription before and after ablation in 100 patients.

months was chosen intentionally as the optimum interval at which to report follow up, to avoid uncertainties about the relevance for longer term outcome of earlier recurrences in patients in persistent AF at the time of ablation. This interval also allows a generous period for atrial reverse remodelling to have occurred and, in our opinion, is likely to provide a truer indication of longer term outcome. ${ }^{3421}$ At each interval during follow up, rhythm status was determined on the basis of symptoms since last review and rhythm on that occasion from a 12 lead ECG. In addition, all patients free of symptomatic AF had a 48 hour Holter ECG recorded to exclude the occurrence of asymptomatic episodes of AF. An echocardiogram was also recorded at most follow up visits to remeasure the size of the left atrium and left ventricular function. Whether antiarrhythmic and anticoagulant treatment was maintained or withdrawn during follow up was decided on the basis of rhythm status and the patient's wishes. If treatment was reduced or withdrawn and symptoms of palpitation recurred or asymptomatic AF was seen on Holter ECGs, treatment was restarted and continued indefinitely.

Table 1 Summary characteristics of the patients and ablation procedure

\begin{tabular}{ll}
\hline Number & 100 \\
Age (years) & 52 (range 23-73) \\
Sex (men:women) & $4: 1$ (range 6-180) \\
AF duration (months) & 53 (range 1-5) \\
Drug failures & \\
AF category at ablation & 36 \\
$\quad$ Paroxysmal & 64 \\
$\quad$ Persistent & 195 (range 130-310) \\
Ablation procedure data & 49 (1-106) \\
$\quad$ Procedure duration (min) & 5999 (range 457-57230) \\
$\quad$ Radiographic screening (min) & 71 \\
$\quad x$ Ray radiation dose (mSv) & 11 \\
Need for cardioversion & 45 \\
Number of PVs ablated & 36 \\
$\quad$ One & 8 \\
Two & \\
Three & Four \\
\hline AF, atrial fibrillation; PVs, pulmonary veins.
\end{tabular}

\section{Statistical methods}

Unless otherwise stated all values are expressed as mean ( $1 \mathrm{SD})$. Groups were compared by $\chi^{2}$ test for discontinuous and Student's $t$ test for continuous variables, adjusted when required for small sample size. Subgroups were compared by analysis of variance. In all cases, differences were considered significant at the $5 \%$ probability level and highly significant at the $1 \%$ probability level.

\section{RESULTS}

One hundred consecutive patients with idiopathic AF underwent their first focal pulmonary vein ablation in the period February 1999 to December 2002. Mean age was 52 years (range 23-73 years) and 81 were men-a four to one male to female preponderance. No patient had sinus node disease or a bradycardia indication for pacing, but one had previously undergone an aortic valve replacement and another surgical division of an accessory pathway. In the years before AF ablation, 33 patients had undergone another form of catheter ablation: right atrial flutter isthmus ablation in 19; right atrial intercaval line ablation in two; both of these right atrial procedures in five; catheter modification of the atrioventricular junction for a separate diagnosis of atrioventricular nodal re-entry tachycardia in two; ablation of the left sided accessory pathway in two; and combined right atrial tachycardia and flutter isthmus ablation in one. Many of

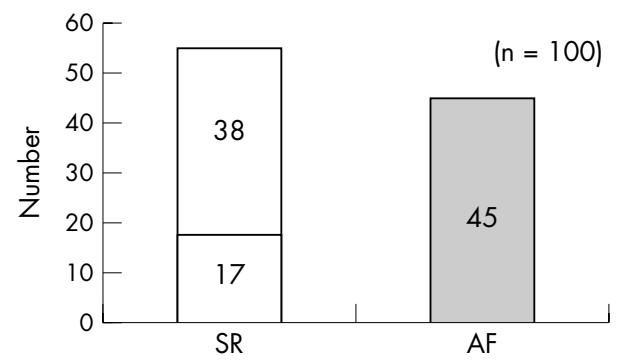

Figure 2 Arrhythmia outcome six months after pulmonary vein ablation. Seventeen patients achieved consistent sinus rhythm (SR) without drugs and 38 with drugs. AF, symptomatic paroxysmal or persistent atrial fibrillation or Holter ECG confirmation of even asymptomatic paroxysmal AF. 
these earlier right atrial ablation procedures were attempts to improve arrhythmia control before the introduction of focal pulmonary vein ablation at this hospital. Two patients had dual chamber pacemakers, capable of anti-AF pacing, implanted previously. No patient had echocardiographic evidence of left ventricular hypertrophy or reduced systolic function, but in 23 the transverse left atrial dimension exceeded the upper limits of normal for this department: $45 \mathrm{~mm}$ in men and $40 \mathrm{~mm}$ in women.

Mean duration of any AF history was 53 months (range 6-180 months) and the primary indication for ablation was uncontrolled symptoms despite drug treatment in 89. The remaining 11 patients underwent ablation in an attempt to avoid long term antiarrhythmic and anticoagulant treatments. Patients took a mean of three (range 1-5) antiarrhythmic drugs before ablation. Figure 1 details antiarrhythmic drug use before and after ablation.

Results of 24 hour Holter ECG recordings were available for 88 patients and AF onset was seen in 58. Initiation was with $\mathrm{P}$ on $\mathrm{T}$ pattern atrial extrasystoles in 31 . Frequent atrial extrasystoles or runs of rapid atrial tachycardia were evident in another four and 10 patients were in AF throughout the recording period. In only 12 patients were the recordings considered normal.

\section{Ablation procedure}

In all but one patient, the procedure was performed under local anaesthesia and midazolam and fentanyl sedation. Although AF had been paroxysmal in all patients at one time in their history, it had become persistent in 64 by the time of their ablation procedure and in only 36 was paroxysmal behaviour maintained. Mean time from initial referral for ablation to actually undergoing the procedure in this series was six months (range 4-9 months). Internal cardioversion was required at least once during the procedure in 71 patients. Table 1 summarises key parameters of the patient cohort and the ablation procedure. In addition, linear ablations were performed with LocaLisa guidance in 19 patients because their AF remained resistant to cardioversion despite pulmonary vein ablation. The introduction of LocaLisa guidance $(n=38)$ reduced total radiographic screening time $(46(124) v 56$ (215) minutes, $\mathrm{p}=0.008)$ and $x$ ray dose $(4806 v 8,249 \mathrm{mSv}, \mathrm{p}=0.04)$ but not total procedure duration $(\mathrm{p}=0.29)$.

\section{Early outcome and discharge management}

At the end of the ablation procedure 77 patients were in sinus rhythm compared with only 22 at the start $(p<0.0001)$. Except for those who experienced complications, patients were discharged the day after the procedure and reviewed regularly thereafter, either at our hospital or at their local

\begin{tabular}{|ll|}
\hline \multicolumn{2}{|l|}{$\begin{array}{l}\text { Table } 2 \text { Complications of focal pulmonary } \\
\text { vein ablation for AF }\end{array}$} \\
\hline Nature of complication & $\begin{array}{l}\text { Complication frequency } \\
\text { (in } 100 \text { procedures) }\end{array}$ \\
\hline Cardiac tamponade* & 6 \\
Coronary artery embolus & 2 \\
Acute LU-PV spasm & 2 \\
Groin haematoma† & 2 \\
Complete heart block & 1 \\
Sedation related hypotension & 1 \\
Upper GI haemorrhage & 1 \\
Symptomatic PV stenosis & None \\
\hline *Requiring pericardiocentesis; trequiring transfusion or \\
fibrin injection of false aneurysm. \\
GI, gastrointestinal tract; LU-PV, left upper pulmonary vein \\
ostium.
\end{tabular}

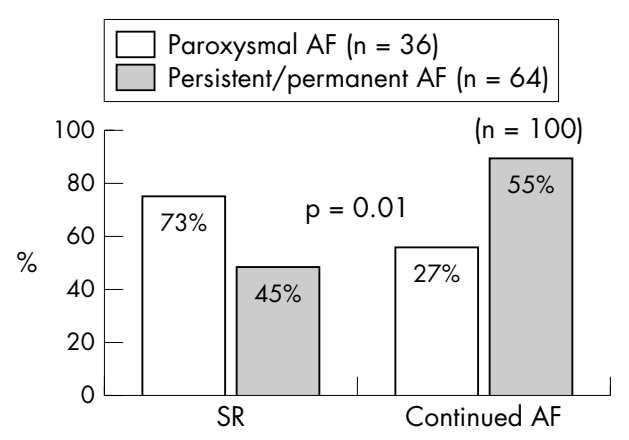

Figure 3 Arrhythmia outcome at six months by AF behaviour at time of ablation.

specialist centre. All those in persistent AF before ablation were prescribed an antiarrhythmic on discharge, typically sotalol, flecainide, or a combination of flecainide and a $\beta$ blocker. All patients with a history of persistent AF restarted full anticoagulation with warfarin and continued for a minimum of three months or until the outcome of the procedure was established by follow up.

\section{Rhythm status six months after ablation}

Figure 2 shows outcome six months after ablation. Of the 100 patients, 55 were consistently in sinus rhythm and free of arrhythmia symptoms. The occurrence of asymptomatic AF was also excluded in these patients by the absence of AF on a 48 hour Holter recording. The remaining 45 patients continued to experience symptomatic paroxysmal or persistent AF despite drug treatment. No patient underwent a repeat ablation procedure during this six month period of follow up, although some have undergone additional procedures subsequently.

Of the 55 patients in stable sinus rhythm after ablation, only $17(31 \%)$ had stopped antiarrhythmic and anticoagulant treatments. The high rate of continuing treatment even in this group was the result of a policy decision to continue antiarrhythmic treatment indefinitely in patients with persistent AF before ablation. This was because asymptomatic runs of AF were detected on Holter recordings of some patients who had persistent AF before the procedure when antiarrhythmic drugs were withdrawn three months after ablation during the first half of the series. Thus, the $69 \%$ rate of continuing treatment to maintain sinus rhythm may not be necessary indefinitely.

\section{Complications of ablation procedure}

Follow up was complete in all patients and none has died or experienced a stroke during or after ablation. Nor have any

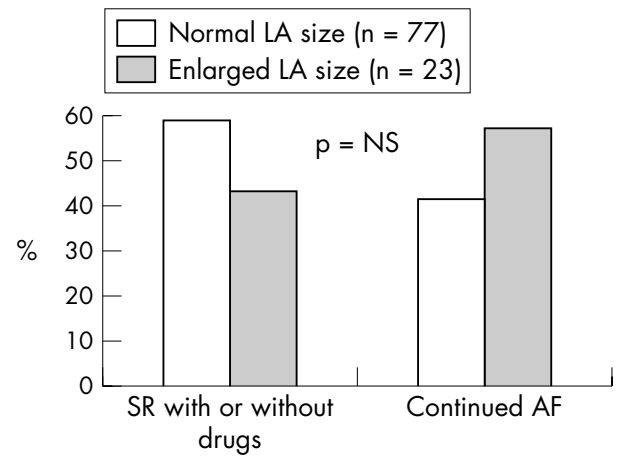

Figure 4 Arrhythmia outcome at six months by left atrial size at time of ablation. LA, transverse left atrial dimension (normal $<40 \mathrm{~mm}$ ); NS, not significant. 

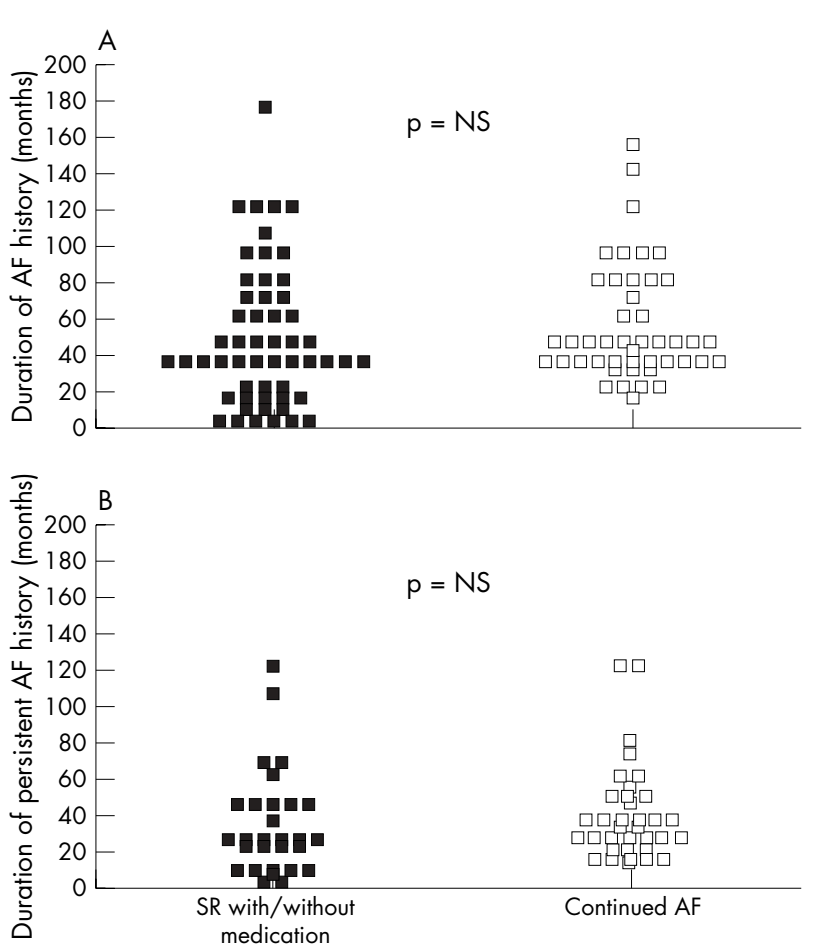

Figure 5 Arrhythmia outcome at six months by (A) duration of $A F$ history and (B) duration of any persistent AF before ablation.

developed symptoms of late pulmonary vein stenosis. However, 12 patients experienced other complications during or shortly after the procedure (table 2 ). Pericardial tamponade resulted in haemodynamic compromise and required pericardiocentesis in six patients. Cardiac perforation was attributed to transseptal puncture in two, to a right ventricular pacing lead in two, to manipulating a stiff cardioversion catheter in the right ventricular outflow tract in one, and to perforation of the right upper pulmonary veins with a guidewire in one. Once cardiac perforation had occurred, bleeding was inevitably aggravated by the need for full anticoagulation throughout the procedure. Each of the two systemic embolic episodes occurred just after transseptal sheath manipulation. On both occasions, the embolus was to the right coronary artery and caused hypotension and ECG changes in the inferior leads, which resolved over a few minutes. No coronary lesions were evident on angiography performed within 10 minutes of the event, and air embolus was presumed to be the cause in both. After a period of observation, the procedure was completed on both occasions without further complication. Complete heart block occurred in one patient during ablation to the right atrial isthmus between the tricuspid valve and inferior vena cava. Local groin haematomas sufficient to require transfusion or fibrin injection to a false aneurysm occurred in two patients and delayed hospital discharge. Acute spasm of the left upper pulmonary vein was observed during the ablation procedure in two patients, but neither developed a significant stenosis on detailed assessment over the next three months. In the whole group, the pulmonary veins were not imaged systematically after the ablation procedure.

\section{Subgroup analyses of outcome of ablation at six months \\ Effect of clinical AF category}

Outcome of ablation at six months correlated significantly with the pattern of AF behaviour at the time of the procedure (fig 3). Thus, the chance of being in sinus rhythm at six months, with or without the use of antiarrhythmic drugs, was $73 \%$ in those with paroxysmal $(n=36)$ as compared with $45 \%$ in those with persistent $\mathrm{AF}(\mathrm{n}=64)$ at the time of ablation $(p=0.01)$. Conversely, the chance of a patient with paroxysmal $\mathrm{AF}$ at the time of ablation continuing to experience $\mathrm{AF}$ at six months was $27 \%$ as compared with $55 \%$ for those with persistent AF (Fisher's exact $\mathrm{p}=0.01$ ). Of patients continuing to have paroxysmal AF at time of ablation and in sinus rhythm consistently six months later $(\mathrm{n}=26), 11(42 \%)$ were no longer taking antiarrhythmic drugs and $15(58 \%)$ were. Of patients in persistent $\mathrm{AF}$ at the time of ablation and in sinus rhythm six months later $(n=29)$, six $(21 \%)$ were no longer taking antiarrhythmic drugs and $23(79 \%)$ were.

\section{Effect of left atrial size}

Outcome of ablation at six months did not relate to whether the transverse diameter of the left atrium on a two dimensional echocardiogram was normal $(n=77)$ or increased $(n=23)$ (Fisher's exact $\mathrm{p}=0.24)$ (fig 4$)$.

Duration of any AF history before ablation

Outcome of ablation at six months did not relate either to an estimate of the total duration of AF history ( sinus rhythm 50.5 (37.9) $v$ AF 56.3 (32.1) months, $\mathrm{p}=0.42$ ) or to the duration of any progression to persistent $\operatorname{AF}(\mathrm{n}=64)$ by the time of the ablation procedure (sinus rhythm 38.1 (28) $v$ AF 38.2 (27.9) months, $\mathrm{p}=0.99$ ) (fig 5).

\section{Effect of patient age on ablation outcome}

Outcome of ablation at six months did not relate to the age of the patient at the time of the procedure (sinus rhythm 51.7 (10.4) v AF 52.1 (10.8) years, $\mathrm{p}=0.87$ ) (fig 6).

\section{DISCUSSION}

The main finding of this study is that $55 \%$ of the first 100 consecutive patients treated by catheter ablation for idiopathic, drug refractory AF at our hospital were in stable sinus rhythm six months later. Although this outcome appears worse than the success rate of $70-90 \%$ reported from pioneering centres in their latest reports, ${ }^{5-7}$ it is similar to the $59-62 \%$ outcome of their initial series ${ }^{2}{ }^{6}$ and well within the $33-66 \%$ success rate reported by other second wave groups. ${ }^{15-17} 22-24$ Furthermore, the results are of outcome after a first pulmonary vein ablation procedure in each patient and not after multiple procedures. ${ }^{23}$ These results also would have included any impact of a learning curve effect in deploying this novel and complex form of ablation. ${ }^{25}$

Retrospective subgroup analyses show that AF was abolished in $73 \%$ of patients continuing to experience paroxysmal AF as compared with only $45 \%$ of patients who had progressed to a persistent pattern before the ablation procedure $(\mathrm{p}<0.01)$. The worse outcome among patients in

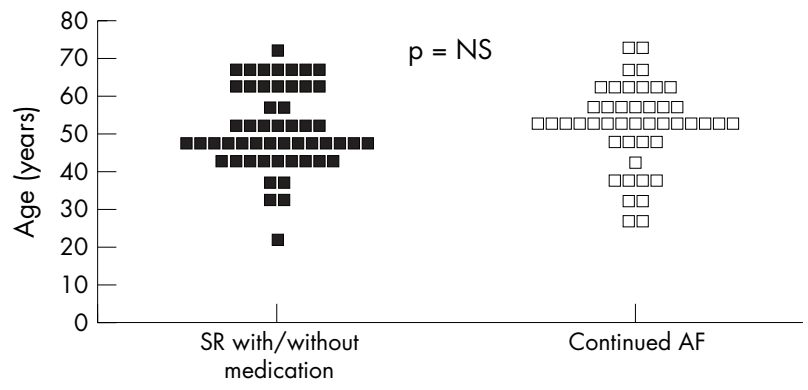

Figure 6 Arrhythmia outcome at six months by patient age at time of ablation. 
persistent $\mathrm{AF}$ at the time of ablation has been highlighted previously by other groups ${ }^{78}$ and correlates with the need for multiple or more extensive procedures in other series. ${ }^{14}{ }^{16}$ The implication of this finding is that, to improve outcome, ablation may need to be undertaken when arrhythmia behaviour is still paroxysmal rather than delaying it further, once optimal drug treatment fails to control symptoms adequately. The fact that the outcome of ablation with current procedures is significantly worse for persistent than for paroxysmal AF affects the risk-benefit equation of the procedure: earlier procedures with higher success versus delayed procedures with lower success rates to avoid exposing patients to procedural risks. Importantly for patient selection, outcome in this series was not influenced by patient age, total duration of any AF history, duration of any persistent AF behaviour, or the transverse dimension of the left atrium.

\section{Differences from other series}

\section{Proportion of patients with persistent AF}

The outcome of any series of AF ablation depends on the case mix of patients studied. In contrast to most other series, in which only patients with paroxysmal $\mathrm{AF}^{251723}$ or only a small proportion with persistent arrhythmias were studied, ${ }^{16}{ }^{26}$ the majority (64\%) in this series had persistent AF. Not only are the results of ablation worse for patients with persisting $\mathrm{AF}$, in which multiple or more extensive procedures may be required, but also antiarrhythmic drugs may need to be prescribed differently during follow up. ${ }^{3}{ }^{21}$ If, as might be expected, the success rate of pulmonary vein ablation falls progressively over time from the onset of persistent AF then linear ablation either alone or in combination with focal pulmonary vein ablation may be more appropriate. ${ }^{27}$ Support for this approach can be extrapolated from surgical experiences; even in the context of structural heart disease, longstanding persistent AF can be abolished in 70-99\% of patients. ${ }^{11-1328} 29$ Similar results would be expected from line drawing procedures performed percutaneously with the aid of equipment designed to facilitate the process.

\section{Continued antiarrhythmic drug treatment after ablation}

Most of the patients in sinus rhythm in this series were still taking potent antiarrhythmic drugs six months after ablation. A policy decision was taken early in the series to continue drugs long term for patients who had persistent AF before ablation. This was adopted after we saw short runs of asymptomatic AF re-emerge on Holter recordings from some of these patients when drug treatment was being withdrawn three months after ablation. In patients with persistent $\mathrm{AF}$, arrhythmia recurrences might still be expected despite ablation of pulmonary vein triggers until atrial remodelling has reversed..$^{24}$ Indeed, our group has already reported the phenomenon of "delayed cure" despite early recurrences of $\mathrm{AF}$ in this context. ${ }^{30}$ For these reasons, the proportion of patients continuing antiarrhythmic treatment in this series indicates reluctance on the part of both patients and physicians to withdraw treatment after ablation, which had been ineffective previously, when control of AF has at last been achieved.

\section{Complications of pulmonary vein ablation}

The debate about the role of catheter ablation of AF is dominated at the moment by concerns about serious complications: cardiac tamponade and thromboembolic stroke acutely and pulmonary vein stenosis in the years afterwards. ${ }^{31}$
Cardiac tamponade occurred in $6 \%$ of patients in this series and, in all but one case, was suspected before the end of the procedure on the basis of falling blood pressure and confirmed by echocardiography. Pericardiocentesis relieved the problem in all, but discharge was delayed by $2-5$ days in every case because of subsequent pericardial discomfort, pyrexia, or malaise in some. As best we can determine, cardiac tamponade was probably not caused primarily by pulmonary vein ablation in any of these cases but followed difficult transseptal procedures or manipulation of a rather stiff cardioversion catheter into the pulmonary artery. The need for anticoagulation subsequently during the procedure then facilitated continued bleeding from any perforation.

Both air emboli to the right coronary arteries occurred just after exchange of a catheter through a transseptal sheath, emphasising again the need for meticulous technique at these times.

To date we have not identified any case of symptomatic late pulmonary vein stenosis. To what extent reports of this complication relate to ablation at more distal sites in pulmonary veins or the use of excessive energy remains unclear. In this series, the energy delivered during each ablation was strictly limiting and lesions were only sited near pulmonary vein ostia. ${ }^{2}{ }^{19}$ Limiting energy delivery in this way is undoubtedly appropriate but may reduce the ability to achieve electrical disconnection of veins with conventional catheters. ${ }^{26}{ }^{32}$

\section{Limitations of the study}

The technique of ablation described in this series can be criticised on at least three counts: for not attempting to ablate all veins in each patient, for not using additional mapping aids in the first 62 patients, and for not confirming pulmonary vein isolation. Each of these could have affected the results.

However, the optimum technique for this form of ablation is still evolving and no single method is established, particularly for patients with persistent AF. ${ }^{33}$ For example, the improved antiarrhythmic outcome following isolation of all pulmonary veins may well be explained by the possibility that even pulmonary veins not causing extrasystoles may facilitate the maintenance of AF once it has started. However, at least during the learning phase of any series, the time devoted to disconnecting veins not manifesting extrasystolic behaviour detracts from that available for dealing with veins that are more obviously implicated in arrhythmia initiation.

In this series we did not attempt to determine systematically whether ablated veins had been isolated electrically from the body of the left atrium, but this is difficult to do in patients continuing in $\mathrm{AF}$ at the end of the procedure. Furthermore, not all groups agree that successful ablation requires complete isolation and, even if complete isolation is desirable, ${ }^{6834}$ it is not acute but long term disconnection that is important. Nor was the quality of life of patients after ablation assessed formally or prospectively in this study.

The conduct of AF ablation at this hospital has already changed in the light of these results. However, despite the recognised shortcomings in our initial series, we consider that the results contribute to the debate about this evolving technique of catheter ablation of AF.

\section{Conclusion}

This series confirms a clinically useful role for catheter ablation in AF. It reinforces the validity of earlier reports and shows that this treatment can be undertaken successfully by non-pioneering groups. However, pulmonary vein ablation is a new and complex form of ablation, arduous for both 
patients and operators alike. At present, it is applicable only to a selected subgroup of patients with highly symptomatic drug resistant AF. As currently performed, AF ablation carries a significant risk of serious complications requiring extensive discussion of the risks and benefits of the procedure beforehand. The longer term impact of ablation on the natural history of AF is unknown and the optimum form of the procedure has yet to be developed. However, for the first time catheter ablation now offers a realistic prospect of achieving stable sinus rhythm in preference to settling for permanent AF or atrioventricular node ablation and permanent ventricular paced rhythm; in that regard, it is another milestone in arrhythmia management.

\section{Authors' affiliations}

J P Bourke, A Dunuwille, D O'Donnell, S Jamieson, S S Furniss, Academic Cardiology Unit, Freeman Hospital, Newcastle upon Tyne, UK

\section{REFERENCES}

1 Blaauw Y, Vann Gelder IC, Criins HJ. Treatment of atrial fibrillation. Heart 2002;88:432-7.

2 Hassaguerre $M$, Jais $P$, Shah DC, et al. Spontaneous initiation of atrial fibrillation by ectopic beats originating in the pulmonary veins. N Engl J Med 1998;339:659-66

3 Allessie M, Ausma J, Schotten U. Electrical, contractile and structural remodelling during atrial fibrillation. Cardiovasc Res 2002;54:230-46

4 Hobbs WJ, Flynn S, Todd DM, et al. Reversal of atrial electrical remodelling after cardioversion of persistent atrial fibrillation in humans. Circulation 2000;101:1145-51.

5 Shah DC, Hassaguerre M, Jais $\mathrm{P}$, et al. Electrophysiologically-guided ablation of the pulmonary veins for the curative treatment of atrial fibrillation. Ann Med 2000;32:408-16.

6 Pappone C, Oreto G, Lambertin F, et al. Catheter ablation of paroxysmal atrial fibrillation using a 3-D mapping system. Circulation 1999;101:1203-8.

7 Hassaguerre $M$, Jais $P$, Shah DC, et al. Electrophysiological end-point for catheter ablation of atrial fibrillation initiated from multiple pulmonary veins. Circulation 2000;101:1409-17.

8 Pappone C, Oreto G, Rosanio S, et al. Atrial electro-anatomic remodelling after circumferential radiofrequency pulmonary vein ablation: efficacy of an anatomic approach in a large cohort of patients with atrial fibrillation. Circulation 2001; 104:2539-44

9 Jais P, Shah DC, Hassaguerre M, et al. Efficacy and safety of septal and leftatrial linear ablation for atrial fibrillation. Am J Cardiol 1999:84:139R-46R.

10 Macle L, Weerasooriya R, Jais $\mathrm{P}$, et al. Radiation exposure during radiofrequency catheter ablation for atrial fibrillation. Pacing Clin Electrophysiol 2003;26:288-91.

11 Sie HT, Beukema WP, Ramdat Misier AR, et al. The radiofrequency modified maze procedure: a less invasive surgical approach to atrial fibrillation during open-heart surgery. Eur J Cardiothorac Surg 2001;19:443-7.

12 Kottkamp H, Hindricks G, Autschbach R, et al. Specific linear left atrial lesions in atrial fibrillation: intraoperative radiofrequency ablation using minimally invasive surgical techniques. J Am Coll Cardiol 2002;40:475-80.

13 Melo J, Adragao P, Neves J, et al. Endocardial and epicardial radiofrequency ablation in the treatment of atrial fibrillation with a new intra-operative device. Eur J Cardiothorac Surg 2000;18:182-6.

14 Kanagaratnam L, Tomassoni G, Schweikert R, et al. Empirical pulmonary vein isolation in patients with chronic atrial fibrillation using a three-dimensional non-fluoroscopic mapping system: long-term follow-up. Pacing Clin Electrophysiol 2001;24:1774-9.

15 Mangrum JM, Mounsey JP, Kok LC, et al. Intracardiac echocardiographyguided, anatomically based radiofrequency ablation of focal atrial fibrillation originating from pulmonary veins. J Am Coll Cardiol 2002;39:1964-72.

16 Deisenhofer I, Schneider MA, Bohlen-Knauf $M$, et al. Circumferential mapping and electrical isolation of pulmonary veins in patients with atrial fibrillation. Am J Cardiol 2003;91:159-63.

17 Sanders P, Morton JB, Deen VR, et al. Immediate and long-term results of radiofrequency ablation of pulmonary vein ectopy for cure of paroxysmal atrial fibrillation using a focal approach. Int Med J 2002;32:202-7.

18 Nakashima H, Kumagai K, Noguchi H, et al. Evaluation of recurrence of atrial fibrillation after pulmonary venous ablation. J Cardiol 2002;40:87-94.

19 Kok LC, Everett TH 4th, Akar JG, et al. Effect of heating on pulmonary veins: how to avoid pulmonary vein stenosis, J Cardiovasc Electrophysiol 2003; 14:250-4.

20 Hocini $M$, Shah DC, Jais $P$, et al. Concealed left pulmonary vein potentials unmasked by left atrial stimulation. Pacing Clin Electrophysiol 2000;23:1832-5

21 Sparks PB, Jayaprakash S, Vohra JK, et al. Electrical remodelling of the atrial associated with paroxysmal and chronic atrial flutter. Circulation 2000; 102:1807-13.

22 Gerstenfeld EP, Guerra P, Sparks PB, et al. Clinical outcome after radiofrequency catheter ablation of focal atrial fibrillation triggers. J Cardiovasc Electrophysiol 2001;12:900-8.

23 Weiss C, Willems S, Risius T, et al. Functional disconnection of arrhythmogenic pulmonary veins in patients with paroxysmal atrial fibrillation guided by combined electro-anatomical (CARTO) and conventional mapping. J Intervent Card Electrophysiol 2002;6:267-75.

24 Stabili G, Bertaglia E, Senatore G, et al. Feasibility of pulmonary vein ostia radiofrequency ablation in patients with atrial fibrillation: a multi-center study (CACAF pilot study). Pacing Clin Electrophysiol 2003;26:284-7.

25 Knight BP, Oral H, Chugh A, et al. Effects of operator experience on the outcome and duration of pulmonary vein isolation procedures for atrial fibrillation. Am J Cardiol 2003;91:673-7.

26 Macle L, Jais P, Weerasooriya R, et al. Irrigated-tip catheter ablation of pulmonary veins for treatment of atrial fibrillation. J Cardiovasc Electrophysiol 2002;13:1067-73

27 Seidl K, Schwacke H, Zahn R, et al. Catheter ablation of chronic atrial fibrillation with non-contact mapping: are continuous linear lesions associated with ablation success? Pacing Clin Electrophysiol 2003:26:534-43.

28 Deneke T, Khargi K, Grewe PH, et al. Left versus bi-atrial Maze operation using intra-operative cooled-tip radiofrequency ablation in patients undergoing open-heart surgery: safety and efficacy. J Am Coll Cardiol 2002:39: 1644-50.

29 Guang Y, Zhen-jie C, Yong LW, et al. Evaluation of clinical treatment of atrial fibrillation associated with rheumatic mitral valve disease by radiofrequency ablation. Eur J Cardiothorac Surg 2002;21:249-54.

30 O'Donnell D, Furniss SS, Dunuwille A, et al. Delayed cure despite early recurrence after pulmonary vein isolation for atrial fibrillation. Am J Cardiol 2003;91:83-5.

31 Arentz T, Jander N, von Rosenthal J, et al. Incidence of pulmonary vein stenosis two years after radiofrequency catheter ablation of refractory atria fibrillation. Eur Heart J 2003:24:963-9.

32 Oral H, Scharf C, Hall B, et al. Catheter ablation for paroxysmal atrial fibrillation: segmental pulmonary ostial ablation versus left atrial ablation. Circulation 2003; 108:2355-60.

33 Marrouche NF, Dresing T, Cole C, et al. Circular mapping and ablation of the pulmonary vein for treatment of atrial fibrillation: impact of different catheter technologies. J Am Coll Cardiol 2002;40:464-74.

34 Stabile G, Turco $P$, La Rocca $V$, et al. Is pulmonary vein isolation necessary for curing atrial fibrillation? Circulation 2003;108:657-60. 